\title{
Robust Optimal Control of Deterministic Information Epidemics with Noisy Transition Rates
}

\author{
Fangzhou Liu*, Zengjie Zhang, Martin Buss ${ }^{1}$ \\ Theresienstr. 90, 80333, Munich, Germany
}

\begin{abstract}
In this paper the robust optimal control of deterministic information epidemics is inspected taking into consideration the noisy transition rates. Distinct from conventional works, the heterogeneous susceptible-infected-susceptible (SIS) model is adopted where both the heterogeneities in the network topology and the individual diversity are considered. In light of the commonly existing noise in the transition processes, we address the robust optimal control problem aiming at maximizing the spreading performance at the finite time instant given a fixed budget. By using the distribution analysis techniques, the inspected problem is transformed to a constrained optimal control problem and solved by the Pontryagin Maximum Principle (PMP). A novel approach combining the forward backward sweep method and the secant method is proposed to efficiently reduce the computation burden. The performance of the robust optimal control as well as the influence of the parameters is examined by numerical experiments in real social networks.
\end{abstract}

Keywords: information epidemics, robust optimal control, noise

\footnotetext{
* Corresponding author

Email address: fangzhou.liu@tum.de (Fangzhou Liu)

${ }^{1}$ F. Liu, Z. Zhang and M. Buss are with the Chair of Automatic Control Engineering, Technical University of Munich; ffangzhou.liu,zengjie.zhang,mb\}@tum.de
}

Preprint submitted to Physica A: Statistical Mechanics and Its ApplicationsNovember 28, 2018 


\section{Introduction}

Information epidemics, which is analogous to epidemics spreading in populations, describes the information dissemination in social networks [1, 2]. Thus the epidemics models are introduced to the field of information diffusion [3, 4, 5].

5 As modeling and control of information diffusion processes draw wider interests in the fields of sociology, psychology, computer science and control [6, 7], information-epidemics-based analysis and controller design has become attractive topics in recent years [8, 9].

Among all the information epidemics models, there exist several common compartments, e.g., susceptible (S) and infected (I). These compartments are naturally connected to the individual states, e.g., awareness and unawareness, or to accept and to refuse in the context of information diffusion. The susceptibleinfected-susceptible (SIS) model [10] is a basic epidemic model, which is widely used in viral marketing and information diffusion processes. The mechanism of

15 the transitions in the SIS model can be described by a Markov chain with $2^{N}$ states, where $N$ is the number of nodes in the network. By using the mean field approximation, a continuous-time node-based SIS model taking into considerations the heterogeneity in networks and diffusion processes is adopted in this article. This model characterizes the dynamics of the approximated infection probability of each individual with the state transition rates as parameters. Nevertheless, noise is inevitable in the diffusion processes in practical situations. In recent works, noise is considered as the behavior of randomly accepting or refusing the information [11] and the individualization force in opinion dynamics [12. Instead of the aforementioned scenarios, the transition processes between different compartments are sensitive to perturbation caused by external noise [13. Whereas this topic is seldom inspected.

Apart from modeling, an attractive topic on information epidemics is to design an optimal control strategy that guides information propagation in complex networks as a desired way [14. According to the recent survey [15], the optimal control design for information epidemics is still an open problem, let alone 
the robust optimal controller design for information epidemics with noise. Although there exist several very recent works regarding optimal control or model predictive control for epidemic models, e.g., [8, 16, none of them considers time-variant control rules. Moreover, to the best of our knowledge, there exist little literature regarding the control of such kind of model with perturbations. To this end, we address the robust optimal control problem for information epidemics with noisy transition rates.

Contribution: In this article, for the first time, a robust optimal control strategy enhancing the information diffusion with perturbed parameters is designed for information epidemics over heterogeneous communication networks. Both the heterogeneities in the network structure and the transition processes are considered by using directed graph description and different transition rates, such that the diversities rooted in the social environment and the individual character are considered. Moreover, the perturbation on the transition rates is 45 introduced, which generally covers most types of uncertainties in information diffusion processes. By manipulating the infection rates, the control input which mimics word-of-mouth is to be designed to robustly maximizing the dissemination. In light of the practical scenarios, the fixed budget constraint is taken into account. Recalling the distribution analysis approach, the inspected problem is transformed into an optimal control problem with a cost function combining the nominal control performance and the influence of the noise. The solution to the proposed problem is achieved taking advantage of the Pontryagin Maximum Principle (PMP). To attain a practically efficient solution, a computationally cheap algorithm combining the forward backward sweep method (FBSM) and the secant method is provided. This result is especially significant for large scale social networks.

The remaining of this article are organized as follows: In Section 2, the node-based SIS model is introduced based on the graph theory. Following the preliminaries, we formulate the robust optimal control problem in Section 3. The 60 solution techniques including the distribution analysis approach and the novel numerical algorithms are presented in detail in Section 4. The simulations in 
Section 5 shows the effectiveness of the proposed control strategy.

\section{Preliminaries}

In this section, the knowledge of graph theory and the node-based SIS model

\subsection{Networks and Graph Theory}

We consider a social network described by a digraph $\mathcal{G}(\mathcal{V}, \mathcal{E}, W)$ with $N$ $(N \geq 2)$ nodes, where $\mathcal{V}=\{1,2, \ldots, N\}$ and $\mathcal{E} \subseteq \mathcal{V} \times \mathcal{V}$ are the sets of nodes and edges, respectively. The terms individual, node, and agent are interchangeably used to avoid ambiguity. The nonnegative matrix $W=\left[w_{i j}\right] \in \mathbb{R}^{N \times N}$ is the adjacency matrix. $w_{i j}>0$ if and only if there exists a link from node $j$ to $i$. In this case, we say node $j$ listens to node $i$ or node $i$ can influence node $j$. Bearing in mind that multitudes of communications like campaigning and news propagation are not mutual but with direction, digraphs are considered throughout this article. To this end, $w_{i j}=w_{j i}$ does not generally hold. It is also assumed that there exists no self loop, i.e., $w_{i i}=0$ and the adjacency matrix is row stochastic, i.e., $\sum_{j=1}^{N} w_{i j}=1, \forall i \in \mathcal{V}$. For the convenience of further presentation, the in-neighborhood of node $i \in \mathcal{V}$ is introduced as

$$
\mathcal{N}_{i}^{i n}=\left\{j: w_{i j}>0, j \in \mathcal{V}\right\}
$$

It is evident that a node $i$ can only obtain information from the members in its in-neighborhood. We confine ourselves that the graphs considered in this article are strongly connected. A digraph is strongly connected if there exist 70 paths between any two vertices.

\subsection{The Node-Based SIS Model}

In this article, the information epidemics is described as the SIS model with heterogeneous transition rates. The SIS epidemic model could be naturally modeled as a Markov chain which possesses two possible states i.e. susceptible

75 (S) and infected (I) [17]. Analogously, in the context of information diffusion, 


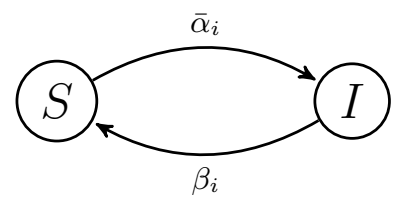

Figure 1: Transitions between different states ( $\mathrm{S}$ and I) of node $i$ with rates $\bar{\alpha}_{i}$ and $\beta_{i}$ in the node-based SIS model.

these two states may refer to awareness and unawareness, respectively. In this article, the infection process (from $\mathrm{S}$ to I) is considered as a proactive action, i.e., each infected individual $i$ infects his/her susceptible social neighbors with rate $\alpha_{i}$ [13]. The curing process (from I to $\mathrm{S}$ ) is assumed to be passive with rates $\beta_{i}$. In light of the diversity of individual character, the transition rates are assumed to be generally different for each agent. This setting as well as the directed communication networks leads to the heterogeneity of the inspected dynamics.

To obtain the node-based SIS model, a mean field approximation approach is utilized. Suppose that all the transition processes for node $i$ are Poisson processes with transition rates $\bar{\alpha}_{i}$ and $\beta_{i}$, where $\bar{\alpha}_{i}$ is calculated based on the infected neighbors of node $i$ as

$$
\bar{\alpha}_{i}=1-\prod_{j=1}^{N}\left(1-\alpha_{j} w_{i j} p_{j}\right) \approx \sum_{j=1}^{N} \alpha_{j} w_{i j} p_{j}, \forall i, j \in \mathcal{V},
$$

where $p_{j}$ is the probability of infection of node $j$. The equal transition map is presented in Figure 1. It follows that the heterogeneous node-based SIS model for node $i$ in a directed network $\mathcal{G}=(\mathcal{V}, \mathcal{E}, W)$ is described as

$$
\dot{p}_{i}=\left(1-p_{i}\right) \sum_{j=1}^{N} \alpha_{j} w_{i j} p_{j}-\beta_{i} p_{i} .
$$

The interpretation of the node-base SIS model (1) is as follows: The change of the infection probability of node $i$ during a time interval $\mathrm{d} t$ consists of two parts, i.e. the influence of the infected neighbors if node $i$ is susceptible; and the curing probability if node $i$ is infected. 
Remark 1. The node-based SIS model (1) is utilized in this article due to its comprehensive physical meaning and far less computational consumption compared with the Markov chain model. Numerical experiments to compare the performance of these two models in undirected graphs have been conducted in [18, which shows the effectiveness of the node-based SIS model.

By denoting $p=\left[p_{1}, \ldots, p_{N}\right]^{\top}, \alpha=\left[\alpha_{1}, \ldots, \alpha_{N}\right]^{\top}$ and $\beta=\left[\beta_{1}, \ldots, \beta_{N}\right]^{\top}$, the compact form of (1) reads

$$
\dot{p}=(I-P) W A p-B p,
$$

where $P=\operatorname{diag}(p), A=\operatorname{diag}(\alpha)$ and $B=\operatorname{diag}(\beta)$. Taking into consideration the noise in the diffusion process, the transition rates in the system (1) are assumed to be perturbed, which is described as

$$
\alpha=\hat{\alpha}+\delta \alpha, \quad \beta=\hat{\beta}+\delta \beta
$$

where $\hat{\alpha}$ and $\hat{\beta}$ are the deterministic nominal transition rates which could be obtained by statistics. $\delta \alpha$ and $\delta \beta$ are the noise reflecting the uncertainties in the transition process. They might be related to the characteristics and decisions of the individuals, as well as the disseminated information, in the social network. For example, to what extent an infected individual would like to share the information to his/her social neighbors is not constant. Without any further investigation to the distribution of the noise, it is natural to assume them as Gaussian Process and independent of each other. Specifically, suppose there holds the following probability density function (PDF)

$$
f_{\text {p.d. }}(\theta)=\frac{1}{(2 \pi)^{N / 2} \operatorname{det}\left(V_{\theta}\right)^{1 / 2}} e^{-\frac{1}{2} \delta \theta^{\top} V_{\theta} \delta \theta}, \theta=\alpha \text { or } \beta,
$$

where $V_{\alpha}, V_{\beta} \in \mathbb{R}^{N \times N}$ are the positive definite covariance matrices. Similar arguments could be referred to in [13]. Based on the node-based SIS model, we are on the way to design the optimal control strategy to enhance the information diffusion. 


\section{Problem formulation}

By interacting with the infection rates, we introduce the control input $u_{i} \in$ $\mathbb{R}$ to each individual, which yields the compact form of the control as $u=$ $\left[u_{1}, \ldots, u_{N}\right]^{\top}$. Thus the controlled information epidemics reads

$$
\dot{p}=(I-P) W(A+U) p-B p,
$$

where $U=\operatorname{diag}(u)$. The control input is required to be limited in the following admissible set

$$
\mathcal{U}=\left\{u: u_{i} \in\left[u_{\min }, u_{\max }\right], \text { Lebesgue integrable, } \forall i \in \mathcal{V}\right\},
$$

where $u_{\min }, u_{\max } \in \mathbb{R}$ are the lower and upper boundaries of the input. Since we consider enhancing the information spreading, it is assumed that $0 \leq u_{\min }<$ $u_{\max }$.

The robust optimal control problem is described as follows.

$$
\begin{gathered}
\min _{u \in \mathcal{U}} J=-\mathbf{1}^{\top} p\left(t_{f}\right), \\
\text { s.t. }(3), p\left(t_{0}\right)=p_{0}, \\
\int_{t_{0}}^{t_{f}} \sum_{i=1}^{N} b_{i}\left(u_{i}\right) \leq \mathcal{B},
\end{gathered}
$$

where $\mathcal{B}>0$ is the fixed budget. $p_{0}$ is the given initial condition, i.e. the probabilities of each people being infected in the very beginning. The term $\left|J\left(t_{f}\right)\right|=\mathbf{1}^{\top} p\left(t_{f}\right)$ describes the (approximated) mathematical expectations of the number of infected people at the fixed terminal time $t_{f}$. The cost function only considers the terminal performance mimics many practical scenarios. For example, in a political campaign nothing counts but the final number of supporters on the voting day. Apart from the cost, $b_{i}(\cdot): \mathbb{R}^{N} \rightarrow \mathbb{R}$ is the consumption function and $\mathcal{B} \in \mathbb{R}$ is the fixed budget, which forms the constraint. It is rationally assumed that $b_{i}(\cdot)$ is continuous, positive and increasing in $u_{i}$. This is based on the fact that the more increment of the infection rate, the more budget is needed as the incentives. Since companies or the campaign teams usually 
have limited budget, the constant $\mathcal{B}$ is introduced as the upper bound for the overtime consumption. It is worth noting that one can calculate the maximum resource needed by substituting $u_{\max }$ into the consumption function. In this paper we only consider the case when $\mathcal{B}<\sum b_{i}\left(u_{\max }\right)$. There is no doubt that the value of $\mathcal{B}$ plays an significant role in the performance of the information diffusion process, which is further illustrated in Section 5. Inspired by the related works e.g., [19, 20], the quadratic form is chosen to model the consumption, i.e., we choose $u^{\top} Q u$ as the consumption function where $Q$ is a constant positive definite diagonal matrix.

Based on the problem formulated in (4), we then provide the solution techniques to calculate the robust optimal control.

\section{Solution to the Robust Control Problem}

To deal with the optimal control for systems with noise, two fundamental ways are commonly used. In the case when the disturbance is deterministic, the optimal control problem can be formulated as zero or non-zero differential games 21. However, it is not suitable for the situation in information epidemics which is perturbed by the noise with stochastic nature, let alone the case in conjugation with the fixed time horizon. An alternative is the stochastic optimal control which takes the expectation as the cost function. Nonetheless, the standard approaches, e.g., solving the Hamilton-Jacobi-Bellman (HJB) equation and utilizing stochastic maximum principle 22], are computationally expensive, especially for systems with large scales which is a general characteristic owned by dynamics on social networks. To this end, a novel approach is provided to solve the problem in (4) approximately. Taking advantage of the property of the dynamics (3), the distribution analysis technique is used to solve the robust optimal control problem. As the fundamental of the proposed method, the optimal control of the nominal information epidemics is first introduced. 


\subsection{Optimal Control of Nominal Information Epidemics}

Before embarking on the robust optimal control problem (4), we investigate the nominal control, i.e., the optimal control of the nominal system. By ignoring the influence of the noise, the nominal system can be obtained as follows

$$
\dot{\hat{p}}=(I-\hat{P}) W(\hat{A}+\hat{U}) \hat{p}-\hat{B} \hat{p},
$$

where the variables and parameters are similarly defined as those in (2). Based on the system (5), the problem in (4) can be rewritten as

$$
\begin{gathered}
\min _{\hat{u} \in \mathcal{U}} \hat{J}=-\mathbf{1}^{\top} \hat{p}\left(t_{f}\right), \\
\text { s.t. }(5), \hat{p}\left(t_{0}\right)=p_{0}, \\
\int_{t_{0}}^{t_{f}} \sum_{i=1}^{N} b_{i}\left(\hat{u}_{i}\right) \leq \mathcal{B} .
\end{gathered}
$$

In order to solve the problem, the isoperimetric constraint in (6) is transformed into the following differential equation

$$
\dot{h}(t):=\hat{u}^{\top}(t) Q \hat{u}(t), h(0)=0, h\left(t_{f}\right)=\mathcal{B} .
$$

Note that the equation of $h(t)$ in (7) is obtained based on the fact that the optimum is unlikely to be achieved unless the budget is sufficiently used. The optimal control problem (11) is then solved based on Pontryagin Maximum Principle (PMP). By introducing the Lagrangian multiplier $\hat{\lambda}_{p} \in \mathbb{R}^{N}$ and $\hat{\sigma} \in \mathbb{R}$, we have the Hamiltonian

$$
\hat{H}=\hat{\lambda}_{p}^{\top}[(I-\hat{P}) W(\hat{A}+\hat{U}) \hat{p}-\hat{B} \hat{p}]+\hat{\sigma} \hat{u}^{\top} Q \hat{u}
$$

According to PMP, we have the dynamics of the costate as follows

$$
\dot{\hat{\lambda}}_{p}=-\frac{\partial \hat{H}}{\partial \hat{p}}=-(\hat{A}+\hat{U}) W^{\top}(I-\hat{P}) \hat{\lambda}_{p}+B \hat{\lambda}_{p}-\hat{\Lambda}_{p} W(\hat{A}+\hat{U}) \hat{p}
$$

140

with the transversality conditions $\hat{\lambda}_{p}\left(t_{f}\right)=\mathbf{- 1}$. Specially, by taking the constraint (7) into consideration, one has $\dot{\hat{\sigma}}=0$, which implies that $\hat{\sigma}$ is a constant but to be fixed. 
By setting $\frac{\partial \hat{H}}{\partial \hat{u}}=0$, we can obtain the control input as follows

$$
\hat{u}^{*}=-\frac{1}{2 \hat{\sigma}^{*}} Q^{-1}\left[\hat{P}^{*} W^{\top}\left(I-\hat{P}^{*}\right) \hat{\lambda}_{p}^{*}\right], \hat{u}^{*} \in \hat{\mathcal{U}}
$$

Although the solution can be analytically presented in the form of $(9)$, the problem in (5) are unlikely to be directly solved following this approach and 145 a numerical solution is necessary. However, inspired by the solution of the nominal information epidemics, the problem in (4) are transformed into a similar formulation in the underlying subsection.

\subsection{Distribution Analysis Approach}

In order to solve the problem in (4), the distribution analysis approach is introduced. It is evident that $p$ is linear in the infection and curing rates, respectively. According to [23], it implies that the covariance of $J(t)=-\mathbf{1}^{\top} p(t)$ can be computed by the covariance of $\alpha$ and $\beta$ as

$$
V_{J}=l_{\alpha}^{\top} V_{\alpha} l_{\alpha}+l_{\beta}^{\top} V_{\beta} l_{\beta},
$$

where

$$
l_{\alpha}^{\top}(t)=\left.\frac{\partial J(t)}{\partial \alpha}\right|_{\alpha=\hat{\alpha}, \beta=\hat{\beta}}, \quad l_{\beta}^{\top}(t)=\left.\frac{\partial J(t)}{\partial \beta}\right|_{\alpha=\hat{\alpha}, \beta=\hat{\beta}} .
$$

Note that the numerator layout notation of the matrix calculus is used. In order to calculate the terminal values of $l_{\alpha}$ and $l_{\beta}$, an additional set of sensitivity equations are integrated in [24]. However, this approach cannot be directly used in (4) because what we consider here is a functional. With slight modification, let $M_{\alpha}:=\frac{\partial p}{\partial \alpha}$ and $M_{\beta}:=\frac{\partial p}{\partial \beta}$. It is clear that $l_{\alpha}=-\left.\mathbf{1}^{\top} M_{\alpha}\right|_{\alpha=\hat{\alpha}, \beta=\hat{\beta}}$ and $l_{\beta}=-\left.\mathbf{1}^{\top} M_{\beta}\right|_{\alpha=\hat{\alpha}, \beta=\hat{\beta}}$. By using the chain rule and the rule for interchanging the order of differentiation for certain mixed partials, the time derivative of $M_{\alpha}$ and $M_{\beta}$ can be obtained as follows

$$
\begin{aligned}
\dot{M}_{\alpha} & =\frac{\partial}{\partial t} M_{\alpha}=\frac{\partial}{\partial t} \frac{\partial p}{\partial \alpha}=\frac{\partial}{\partial \alpha} \dot{p} \\
& =\frac{\partial}{\partial \alpha}((I-P) W(A+U) p-B p)+\mathscr{J} \frac{\partial p}{\partial \alpha} \\
& =(I-P) W P+\mathscr{J} M_{\alpha} \\
\dot{M}_{\beta} & =-P+\mathscr{J} M_{\beta},
\end{aligned}
$$


where $\mathscr{J}$ is the Jacobian matrix and reads,

$$
\mathscr{J}=\frac{\partial \dot{p}}{\partial p}=(I-P) W(A+U)-\operatorname{diag}(W(A+U) p)-B .
$$

To this end, the minimization of $J$ in (4) can be approximated by the combination of the following two parts

$$
J_{1}=-\mathbf{1}^{\top} \hat{p}\left(t_{f}\right), \quad J_{2}=V_{J}\left(t_{f}\right)
$$

where $J_{1}$ is the nominal cost function with respect to the controlled nominal system (5) and $J_{2}$ is the variance of $J$ around the nominal value caused by the parameter uncertainty. Note that by introducing the auxiliary systems (10), the problem with noise in (4) is transformed into the optimal control problem in deterministic systems. Rather than formulating a multi-objective problem of $\left[J_{1}, J_{2}\right]$, we introduce the weighted sum of $J_{1}$ and $J_{2}$, which yields the following optimal control problem.

$$
\begin{aligned}
& \min _{u \in \mathcal{U}} J_{1}+r J_{2}, \\
& \text { s.t. }(5), \quad 10), \hat{p}\left(t_{0}\right)=p_{0}, \\
& \int_{t_{0}}^{t_{f}} u^{\top} Q u \leq \mathcal{B}
\end{aligned}
$$

where $r>0$ is the weighting coefficient. It is worth noting that as a trade-off between the desired cost $J_{1}$ and the influence of the noise $J_{2}$, the value of $r$ is of great significance in (11). Generally, $r$ is set in advance by the companies or the campaign teams based on their knowledge or prediction of the noise. Specifically, smaller $r$ infers that the influence of noise is not considered as very important and vice versa. Further discussions are given in Section 5 to provide valuable insights on the selection of $r$.

Remark 2. By doing the distributional analysis, the robust optimal control problem in (4) is transformed into the optimal control problem in (11). As a sacrifice, the dynamics are augmented with $2 N$ vector differential equations in 100 . To deal with this problem, the simultaneous corrector method is proposed in 25] to solve this large-scale problem in an efficient manner. Bearing 
in mind the following solution to the optimal control problem, an alternative approach is used which is presented in detail in the last part of this section.

The optimal control problem (11) is then solved based on PMP. As a prior step, the dynamics in 10 should be reshaped in a vector form. By denoting $m_{\alpha, i}$ the $i$ th column of $\left.M_{\alpha}\right|_{\alpha=\hat{\alpha}, \beta=\hat{\beta}}, \forall i \in \mathcal{V}$ and similarly for $m_{\beta, i}$, one has

$$
\begin{aligned}
& \dot{m}_{\alpha, i}=\hat{p}_{i}(I-\hat{P}) W_{i}+\mathscr{J} m_{\alpha, i} \\
& \dot{m}_{\beta, i}=-\hat{p}_{i} \mathbf{e}_{i}+\mathscr{J} m_{\beta, i}, \forall i \in \mathcal{V},
\end{aligned}
$$

where $\mathbf{e}_{i} \in \mathbb{R}^{N}$ is the $i$ th column of the unity matrix and $W_{i}$ is the $i$ th column of the adjacency matrix $W$.

By introducing the Lagrangian multiplier $\lambda_{p}, \lambda_{\alpha, i}, \lambda_{\beta, i} \in \mathbb{R}^{N}, \forall i \in \mathcal{V}$ and $\sigma \in \mathbb{R}$, we have the Hamiltonian

$$
\begin{aligned}
H & =\lambda_{p}^{\top}[(I-\hat{P}) W(\hat{A}+U) \hat{p}-\hat{B} \hat{p}]+\sigma u^{\top} Q u \\
& +\sum_{i=1}^{N} \lambda_{\alpha, i}^{\top}\left[\hat{p}_{i}(I-\hat{P}) W_{i}+\mathscr{J} m_{\alpha, i}\right]+\lambda_{\beta, i}^{\top}\left[-p_{i} \mathbf{e}_{i}+\mathscr{J} m_{\beta, i}\right] .
\end{aligned}
$$

According to PMP, the time-derivatives of the costates read

$$
\begin{aligned}
\dot{\lambda}_{p} & =-\frac{\partial H}{\partial \hat{p}}=-(\hat{A}+U) W^{\top}(I-\hat{P}) \lambda_{p}+B \lambda_{p}-\Lambda_{p} W(\hat{A}+U) \hat{p} \\
& +\sum_{i=1}^{N} \hat{p}_{i} \operatorname{diag}\left(W_{i}\right) \lambda_{\alpha, i}-\mathbf{e}_{i} \lambda_{\alpha, i}^{\top}(I-\hat{P}) W_{i}+\operatorname{diag}\left(\mathbf{e}_{i}\right) \lambda_{\beta, i} \\
& +\sum_{i=1}^{N} \Lambda_{\alpha, i} W(\hat{A}+U) m_{\alpha, i}+\Lambda_{\beta, i} W(\hat{A}+U) m_{\beta, i} \\
& +(\hat{A}+U) W^{\top}\left(M_{\alpha, i} \lambda_{\alpha, i}+M_{\beta, i} \lambda_{\beta, i}\right), \\
\dot{\lambda}_{\alpha, i} & =-\frac{\partial H}{\partial m_{\alpha, i}}=-\mathscr{J}^{\top} \lambda_{\alpha, i}, \quad \dot{\lambda}_{\beta, i}=-\frac{\partial H}{\partial m_{\beta, i}}=-\mathscr{J}^{\top} \lambda_{\beta, i}
\end{aligned}
$$

with the transversality conditions

$$
\begin{aligned}
\lambda_{p}\left(t_{f}\right) & =-\mathbf{1}, \lambda_{\alpha, i}\left(t_{f}\right)=-2 r\left(\mathbf{1}^{\top} \otimes \mathbf{e}_{i}\right) V_{\alpha} M_{\alpha}\left(t_{f}\right) \mathbf{1}, \\
\lambda_{\beta, i}\left(t_{f}\right) & =-2 r\left(\mathbf{1}^{\top} \otimes \mathbf{e}_{i}\right) V_{\beta} M_{\beta}\left(t_{f}\right) \mathbf{1}, \quad \forall i \in \mathcal{V} .
\end{aligned}
$$

165 Specially, in light of the constraint (7), one has $\dot{\sigma}=0$, which implies that $\sigma$ is a constant but to be fixed. 
By setting $\frac{\partial H}{\partial u}=0$, we can obtain the control input as follows

$$
\begin{aligned}
u^{*} & =-\frac{1}{2 \sigma^{*}} Q^{-1}\left[\hat{P}^{*} W^{\top}\left(I-\hat{P}^{*}\right) \lambda_{p}^{*}+\sum_{i=1}^{N} M_{\alpha, i} W^{\top}(I-P) \lambda_{\alpha, i}\right. \\
& \left.+M_{\beta, i} W^{\top}(I-P) \lambda_{\beta, i}-P W^{\top}\left(M_{\alpha, i} \lambda_{\alpha, i}+M_{\beta, i} \lambda_{\beta, i}\right)\right], u^{*} \in \mathcal{U} .
\end{aligned}
$$

Remark 3. On one hand, the terms in the dynamics of costates (8) and the input (9) of the nominal system are reserved in (12) and (13), respectively. On the other hand, the distinguishing terms shows the influence of the noise. To this end, the distribution analysis approach reveals the consistency associated with the nominal optimal control solution.

As with the control in (9), $u^{*}$ in $(13)$ cannot be directly calculated due to the impossibility of attaining the optimum in advance. Thus a novel algorithm is proposed to obtain the control input numerically, which is also applicable to the problem (6) with slight modification.

\subsection{Solution Techniques}

As a commonly adopted approach, the shooting method is used in [19]. However, in that case, the arbitrary initial condition of a scalar costate is hard to choose. Taking advantage of the fact that $\sigma$ is a constant scalar, an alternative way to obtain its value becomes a natural idea to solve the problem in (11). Conventionally, the value of $\sigma$ is obtained by trial-and-error [20] which is technically difficult and time-consuming to implement because a sufficiently small initial guess is needed and no efficient update law is given. As a modification, the secant method has been reported to be implemented to solve a similar problem in [26]. However, the issue tacked there is of low dimension and the algorithm is not precisely presented. Inspired by the previous works, we provide the a novel algorithm systematically. Apart from the secant method to search the value of $\sigma$, the forward backward sweep method (FBSM) is also utilized to calculate the control input iteratively. Thus we provide the combined approach as in Algorithm 1.

Apparently, once the costate $\sigma$ is given, one can obtain a cost according to the consumption function. It implies that there exists a map error $=\mathcal{B}-f(\sigma)$ 


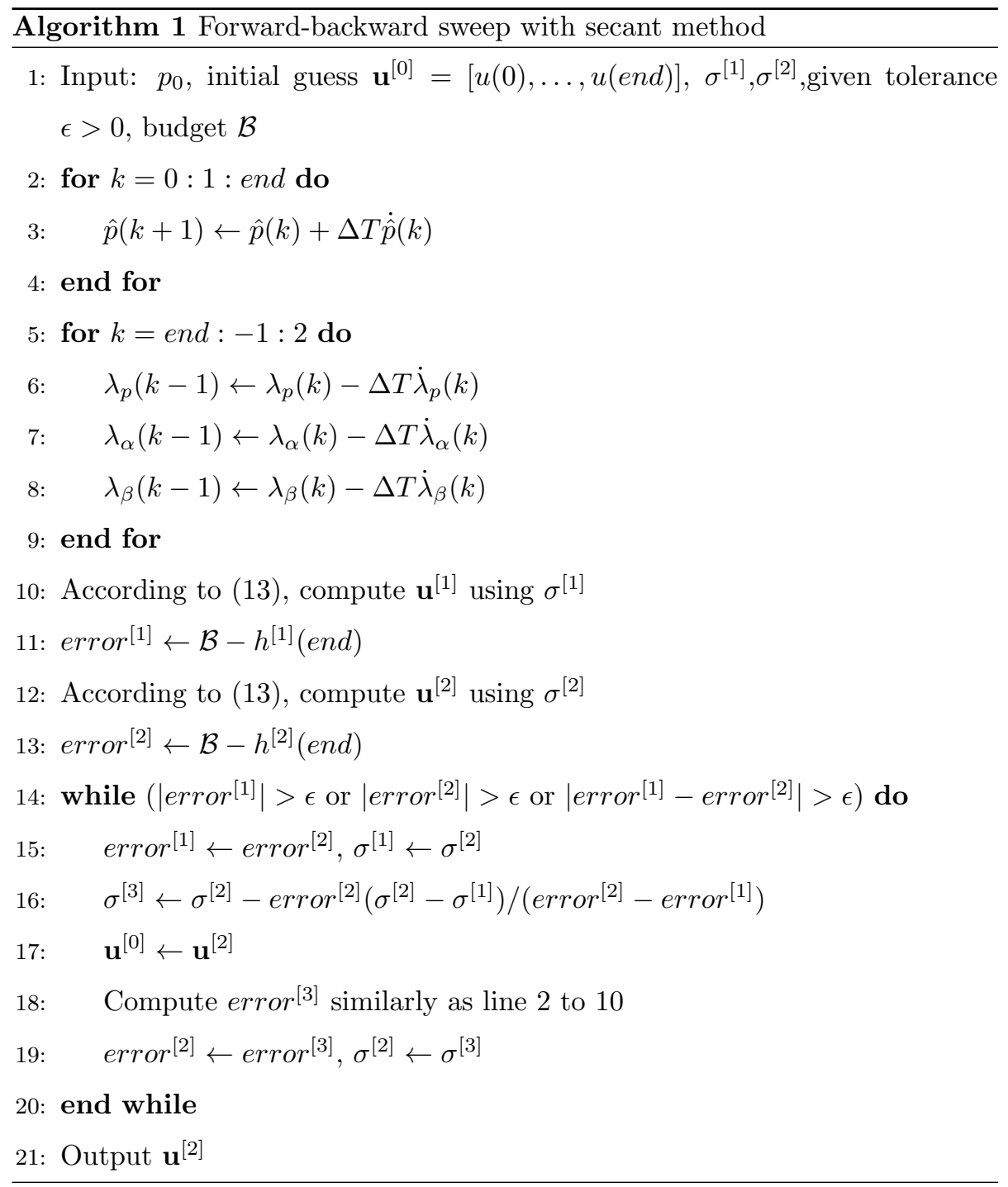


where $f(\cdot): \mathbb{R} \rightarrow \mathbb{R}$ is a function of $\sigma$. It yields that $\sigma$ is the root when the error equals zero. Based on this fact, the FBSM and the secant method can be combined together. Given the initial condition of $p$ and initial guess of the control input, the states overtime can be obtained by forwardly implementing the dynamics (5). As a successive step, the costates can be computed backwardly. By utilizing the errors between the budget and the consumption resulted from two initial values of $\sigma$, the secant method can be applied to update this constant costate. This iteration ends when the errors reach certain tolerance. The convergence analysis of the FBSM can be referred to in [27] and is saved for triviality. Compared with conventional optimization algorithms, e.g., Matlab fmincon, the proposed approach is computationally much cheaper and also capable of dealing with high dimensional problems. Note that the proposed algorithm is able to be applied to the problem in (6) by replacing the respective equations of costates in lines 6 to 8 by the ones in (8). The detailed discussions are presented in Section 5 .

\section{Numerical Experiments}

In this section, the performance of the node-based SIS model under robust optimal control (4) is examined to show the effectiveness of the designed control strategy.

We inspect the information epidemics on two real networks with slight modification. The first network describes the friendship between boys in a small highschool in Illinois [28] (referred as the highschool network), whose largest strongly connected subgraph containing 67 nodes is utilized. The second network shows the friendship between the residents living at a residence hall located on the Australian National University campus 29] (referred as the residence hall network). Similarly, a 214-node strongly connected subgraph is extracted. The weights of the in-edges are normalized such that the adjacency matrix is row stochastic. The effectiveness of the proposed robust optimal control and its comparison with the nominal control are examined in both networks such that 
the results are convincing. The nominal transition rates $\hat{\alpha}_{i}$ and $\hat{\beta}_{i}$ are randomly chosen in the intervals $(0.05,0.15)$ and $(0.005,0.015)$, respectively. This guarantees the heterogeneity of the SIS model. The covariance matrices $V_{\alpha}$ and $V_{\beta}$ are selected to be diagonally dominant and their diagonal entries are randomly chosen within $(0.3,0.35)$. The diagonal weighting matrix $Q$ is selected according to the cardinality of each node, i.e., the $i$ th diagonal entry $q_{i}=0.1\left|\mathcal{N}_{i}^{i n}\right|$, which mimics the natural fact that more influential one individual is the more incentives should be paid to gain his/her help for information spreading.

The initial condition $p_{i}(0)$ is a scalar in $[0,0.01)$ for all $i \in \mathcal{V}$. Note that there are around $20 \%$ of the nodes with initial value zero. These configurations are valid for all the simulations in this section. During all the simulations, the information epidemics is discretized with the sampling period $\Delta T=0.01$. Other fixed parameters are the initial time instant $t_{0}=0, u_{\min }=0$ and $u_{\max }=0.5$.

The validation of the proposed robust optimal control is first conducted by comparing with the system under no control and heuristic control on the highschool network. By choosing the budget $\mathcal{B}=30, t_{f}=5$, the robust optimal control can be calculated by Algorithm 1. Note that according to 24], the initial conditions for $m_{\alpha, i}$ and $m_{\beta, i}$ are both set to $\mathbf{0}$. To make an evaluable comparison, a heuristic control is implemented such that the control input is identical for each node at every time instance. In light of the consumption function and the given budget $\mathcal{B}$, this control input equals 0.4088 entry-wise. The simulation results of the expectation of the number of infected people overtime $(|J(t)|)$ are shown in Figure 2 A similar numerical experiment is conducted on the residence hall network. We set the budget as $\mathcal{B}=30$ while other parameters remain unchanged. The respective heuristic control is 0.2602. The results are presented in Figure 3. It is evident that the controlled epidemics, both the heuristic and the robust optimal control, shows better performance, since the diffusion processes are extraordinarily enhanced compared with the uncontrolled. Apart from that, the proposed robust optimal control scheme reveals a superior performance in terms of $|J(t)|$. It is worth noting that this performance is achieved by making full use of the budget over the fixed control 
horizon which implies that the constraints are fulfilled.

We then compare the performance of the dynamics under robust control obtained by solving (11) with that of the nominal control using the same configuration as in the first simulations. The control inputs are calculated offline: the robust control is obtained by solving (11) while the nominal control by solving (4) but with respect to the nominal system (5). The simulations on both networks are conducted 1,000 times, each of which contains noise in both infection and curing rates. By taking the differences in $\mathbf{1}^{\mathrm{\top}} p\left(t_{f}\right)\left(\left|J\left(t_{f}\right)\right|\right)$ as the index, the performance is shown in Figure 4. For the highschool network (Figure 4 (a) and (b)) and the residence hall network (Figure 4 (c) and (d)), almost all the simulations show that the robust optimal control results in slightly better performance than the nominal control. It implies that under this configuration, the information spreads generally wider under the robust control. Although the differences are not notably large, T-test for both scenario shows a two-tailed $p$-value far less than 0.001 , which means that the results are significantly different and the results in Figure 4 are convincing. Note that if the control input is dominant compared with the transition rates and social networks with larger scale are considered, better performance can be expected.

Based on the problem formulation and the proposed solution technique, the weight $r$ and the budget $\mathcal{B}$ are two of the most influential factors. Specifically, $r$ determines the trade-off between the real objective and the influence of the noise and $\mathcal{B}$ stands for the upper bound of resources. By choosing $r$ from 0 to 1 with step 0.1 and $\mathcal{B}$ from 15 to 35 with step 5 , we compare $\left|J\left(t_{f}\right)\right|$ under each settings on the highschool network. Other configurations are the same with those in the first simulation. As is presented in Figure 5 , it is evident that for the same weight $r$, more budget results in better spreading performance. It is worth noting that the increment of $\left|J\left(t_{f}\right)\right|$ slows down as the budget increases. This yields that companies and campaign teams should wisely plan their budget to reach a balance between the resource and objective. As for the case when the budget is fixed, the performance of $\left|J\left(t_{f}\right)\right|$ fluctuate with respect to the change of $r$. Taking $\mathcal{B}=20$ and 35 as examples, $\left|J\left(t_{f}\right)\right|$ reaches the peak when $r=0.1$ 


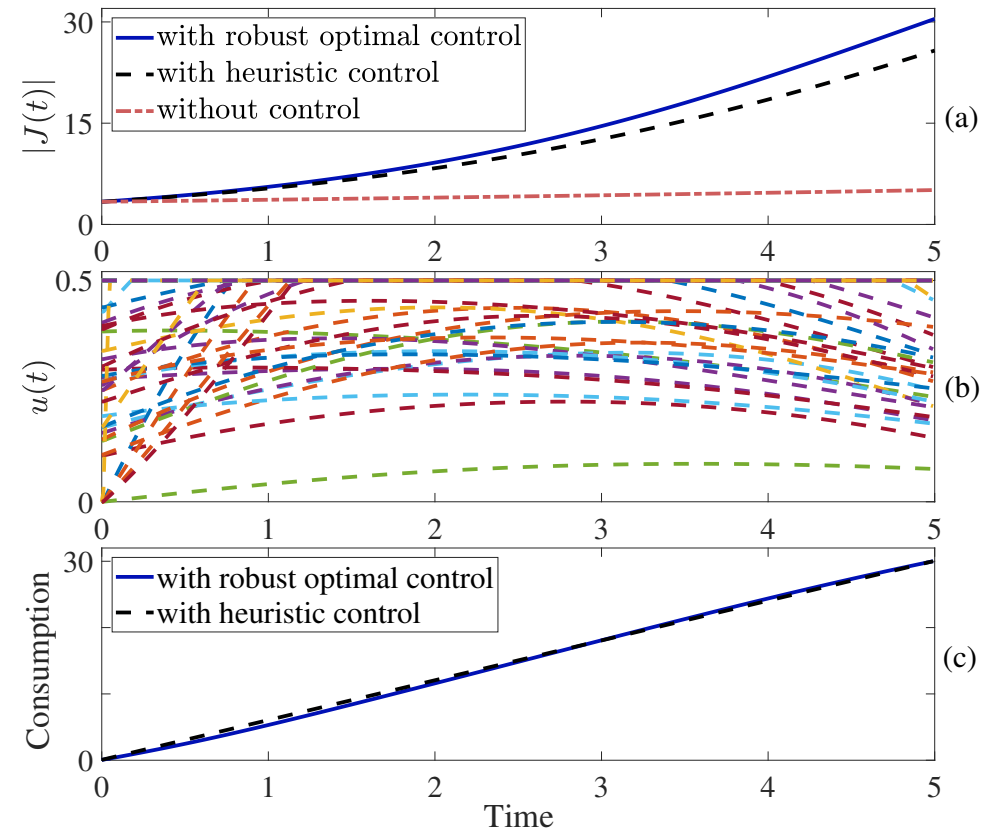

Figure 2: The performance of the diffusion process on the highschool network with 67 nodes. (a) Comparison of $|J(t)|$ with no control, with heuristic control (identical control input, 0.4088, for each node at each time instance) and with robust optimal control. The robust optimal controller shows better performance than the heuristic control and uncontrolled epidemics. (b) The control input of the robust optimal control for each node, which is bounded in $\left[u_{\min }, u_{\max }\right]$. (c) The consumption over time. For both heuristic control and robust optimal control, the budget is adequately used. The initial condition is set as $p_{i}(0) \in[0,0.01)$. Other parameters are: $\hat{\alpha} \in(0.05,0.15), \hat{\beta} \in(0.005,0.015), \mathcal{B}=30, t_{0}=0, t_{f}=5, u_{\min }=0$ and $u_{\max }=0.5$. 

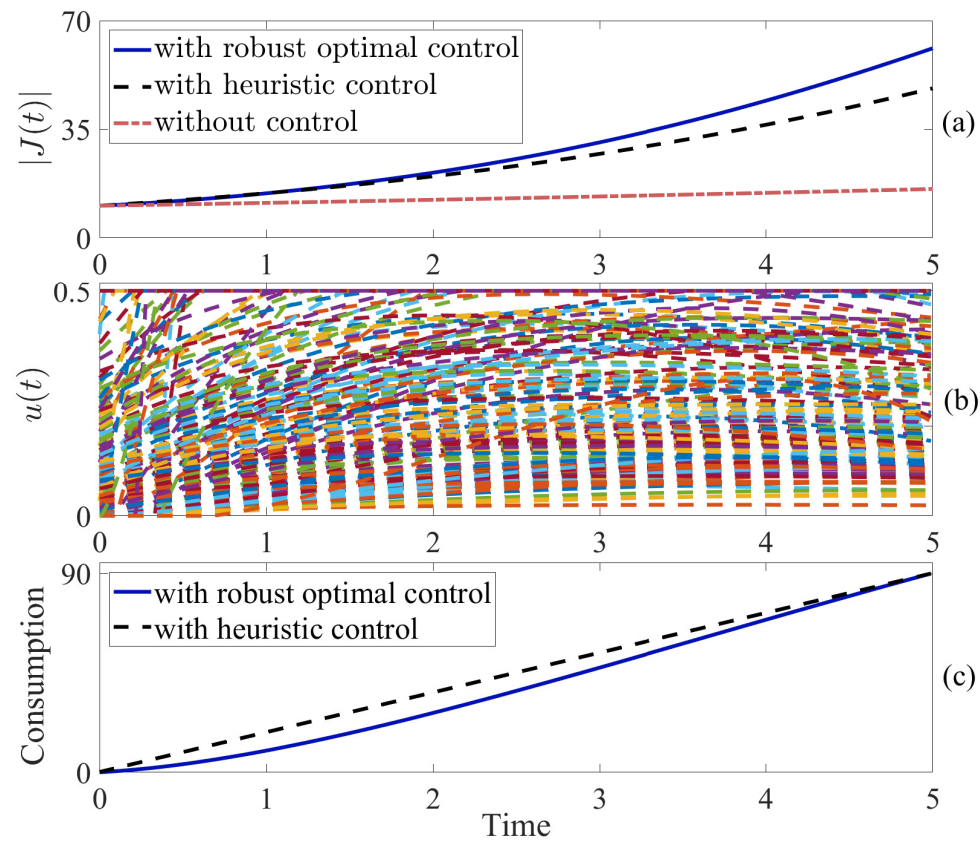

Figure 3: This simulation is conducted over the residence hall network with 214 nodes. The initial condition is set as $p_{i}(0) \in[0,0.01)$. Other parameters are chosen as: $\hat{\alpha} \in(0.05,0.15)$, $\hat{\beta} \in(0.005,0.015), \mathcal{B}=90, t_{0}=0, t_{f}=5, u_{\min }=0$ and $u_{\max }=0.5$. (a) The performance of the diffusion process with no control, with heuristic control (identical control input, 0.2602, for each node at each time instance) and with optimal control is compared in the form of $|J(t)|$. The robust optimal controller shows better performance than the heuristic control and uncontrolled epidemics. (b) The control input of the optimal control for each node, which is bounded in $\left[u_{\min }, u_{\max }\right]$ (c) The consumption over time. For both heuristic control and robust optimal control, the budget is adequately used. 


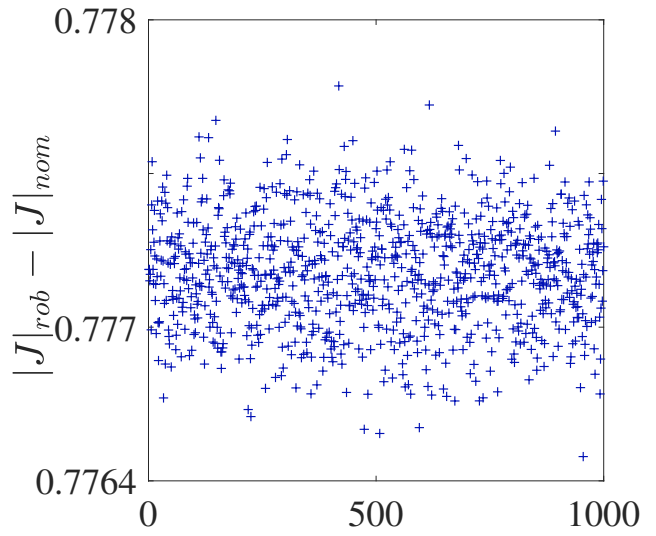

(a)

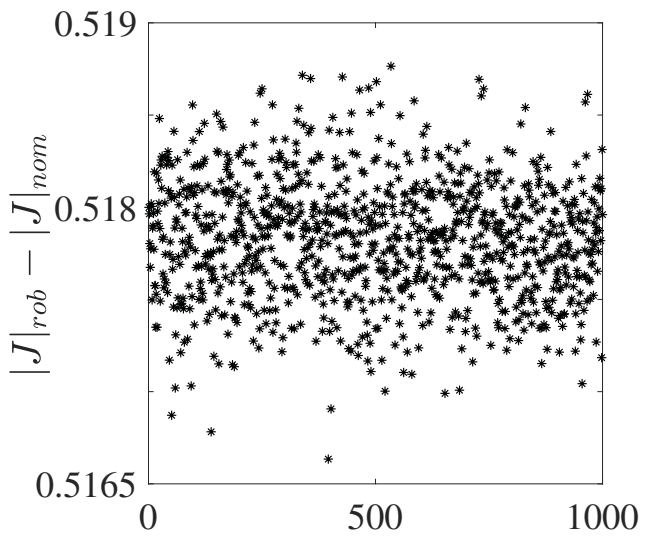

(c)

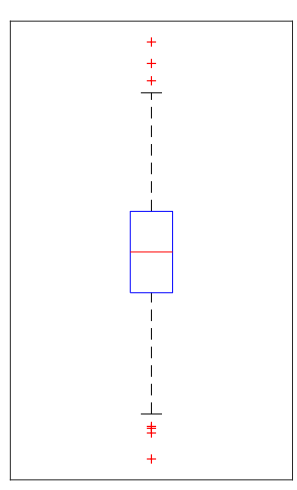

(b)

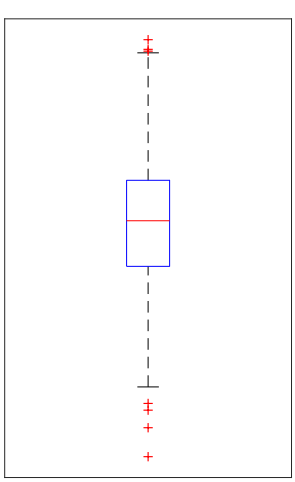

(d)

Figure 4: Comparison between robust optimal control and nominal control in the form of $|J|_{\text {rob }}-|J|_{\text {nom }}$ on highschool network and residence hall network with 1000 runs. (a) and (b) are the scatter plot and box plot of the index for the scenario on the highschool network, respectively. (c) and (d) are for the scenario on the residence hall network. In both cases, the robust optimal control results in slightly better performance than the nominal control. 
and 0.2 , respectively. To this end, the choice of $r$ is nontrivial which may highly

of the affordable impact of the noise are necessary.

\section{Conclusion}

In this paper we address the robust optimal control problem for information epidemics in a heterogeneous network with noisy transition rates. Thus for the first time, the effects of natural uncertainties of the transition rates are considered to determine a robust optimal control strategy. A numerical solution is obtained based on distribution analysis. The diffusion is maximized in finite horizon with the proposed control strategy allowing for the constraint of limited budget. The proposed algorithm, which combines the forward backward sweep method and the secant method, shows its effectiveness and efficiency in dealing with the diffusion processes over real networks. The numerical experiments on the influence of parameters confirm the common sense that the more budget the better dissemination performance. Apart from that, it is of great significance to properly weigh the impact of the noise while utilizing the proposed approach. The addressed formulation and the proposed optimal strategy not only solve the specific problem caused by the noisy transition rates but also provide a general solution to social networks with stochastic perturbations.

Future works should also focus on the distributed control problem and drivenode selection in information epidemics.

\section{References}

[1] W. Goffman, V. Newill, Generalization of epidemic theory, Nature 204 (4955) (1964) 225-228.

[2] M. Li, X. Wang, K. Gao, S. Zhang, A survey on information diffusion in online social networks: Models and methods, Information 8 (118) (2017) 


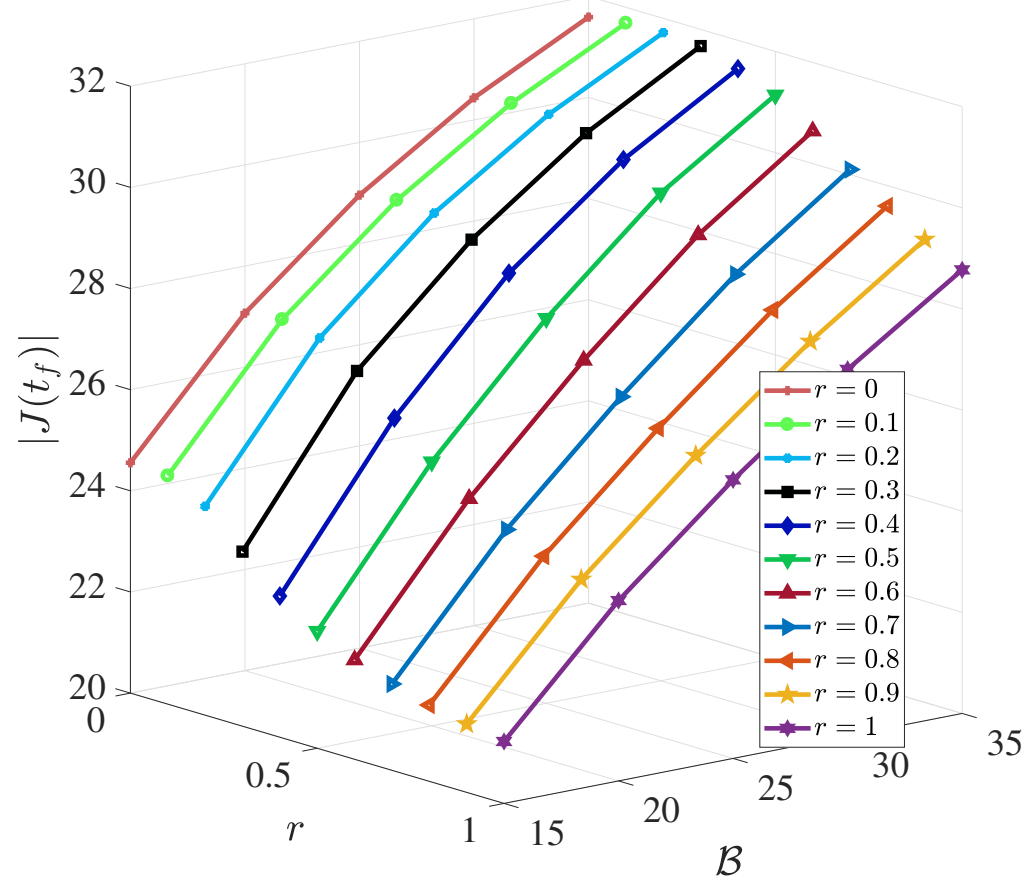

Figure 5: $\left|J\left(t_{f}\right)\right|$ under different configuration of $r$ and $\mathcal{B}$. The parameter $r$ is chosen from 0 to 1 with step 0.1 and $\mathcal{B}$ from 15 to 30 with step 5 . The control input is computed by Algorithm 1 with each pair of the parameters. The initial condition $p_{i}(0)$ is chosen in $[0,0.01)$ and is identical for every experiment. It reveals that more budget results in better spreading performance whereas the choice of $r$ is nontrivial which may depends on the knowledge of noise. 
[3] X. Rui, F. Meng, Z. Wang, G. Yuan, C. Du, Spir: The potential spreaders involved sir model for information diffusion in social networks, Physica A: Statistical Mechanics and its Applications 506 (2008) 254-269.

[4] Y. Pan, Z. Yan, The impact of multiple information on coupled awarenessepidemic dynamics in multiplex networks, Physica A: Statistical Mechanics and its Applications 491 (2018) 45-54.

[5] Y. Liu, S.-M. Diao, Y.-X. Zhu, Q. Liu, Shir competitive information diffusion model for online social media, Physica A: Statistical Mechanics and its Applications 461 (2016) 543-553.

[6] C. Chamley, A. Scaglione, L. Li, Models for the diffusion of beliefs in social networks: An overview, IEEE Singal Processing Magazine 30 (3) (2013) $16-29$.

[7] C. Wang, Z. Tan, Y. Ye, L. Wang, K. H. Cheong, N. gang Xie, A rumor spreading model based on information entropy, Scientific Report 7 (9615).

[8] C. Nowzari, V. M. Preciado, G. J. Papas, Optimal resource allocation for control of networked epidemic models, IEEE Transactions on Control of Network Systems 4 (2) (2017) 159-169.

[9] S. Han, V. M. Preciado, C. Nowzari, G. J. Pappas, Data-driven network resource allocation for controlling spreading processes, IEEE Transactions on Network Science and Engineering 2 (4) (2015) 127-138.

[10] W. O. Kermack, A. G. McKendrick, A contribution to the mathematical theory of epidemics, Proc. Roy. Soc. A 115 (772) (1927) 700-721.

[11] M. T. Gastner, N. Markou, G. Pruessner, M. Draief, Opinion formation models on a gradient, PLoS ONE 23 (12) (2014) e114088.

335 [12] N. Askitas, Explaining opinion polarisation with opinion copulas, PLoS ONE 12 (8) (2017) e0183277. 
[13] B. Qu, H. Wang, Sis epidemic spreading with heterogeneous infection rates, IEEE Transactions on Network Science and Engineering 4 (3) (2017) 177186.

[14] F. Liu, M. Buss, Optimal control for information diffusion over heterogeneous networks, in: 55th IEEE Conference on Decision and Control (CDC), 2016, pp. 141-146.

[15] C. Nowzari, V. M. Preciado, G. J. Pappas, Analysis and control of epidemics: A survey of spreading processes on complex networks, IEEE Control Systems 36 (1) (2016) 26-46.

[16] M. Ogura, V. M. Preciado, Efficient containment of exact sir markovian processes on networks, in: 55th IEEE Conference on Decision and Control, 2016, pp. 967-972.

[17] P. V. Mieghem, J. Omic, R. Kooij, Virus spread in networks, IEEE/ACM Transactions on Networking 17 (1) (2009) 1-14.

[18] P. E. Paré, C. L. Beck, A. Nedić, Epidemic processes over time-varying networks, IEEE Transactions on Control of Networked Systems. In press.

[19] K. Kandhway, J. Kuri, How to run a campaign: Optimal control of sis and sir information epidemics, Applied Mathematics and Computation 231 (2014) 79-92.

[20] R. M. Neilan, S. Lenhart, Dimacs series in discrete mathematics and theoretical computer science, International Journal of Computer Applications 75 (2010) 67-81.

[21] F. Lewis, D. Vrabie, V. Syrmos, Optimal Control, John Wiley \& Sons, Inc., 2012.

[22] S. Peng, A general stochastic maximum principle for optimal control problems, SIAM Journal of Control and Optimization 28 (4) (1990) 966-979. 
[23] Z. K. Nagy, R. D. Braatz, Open-loop and closed-loop robust optimal control of batch processes using distributional and worst-case analysis, Journal of Process Control 14 (4) (2004) 411-422.

[24] R. P.Dickinson, R. J.Gelinas, Sensitivity analysis of ordinary differential equation systems - a direct method, Journal of Computational Physics 21 (2) (1976) 123-143.

[25] W. F. Feehery, J. E. Tolsma, P. I. Barton, Efficient sensitivity analysis of large-scale differential-algebraic systems, Appl. Numer. Math. 25 (1) (1997) $41-54$.

[26] A. Hamdache, I. Elmouki, S. Saadi, Optimal control with an isoperimetric constraint applied to cancer immunotherapy, International Journal of Computer Applications 94 (15) (2014) 31-37.

375 [27] M. McAsey, L. Mou, W. Han, Convergence of the forward-backward sweep method in optimal control, Computational Optimization and Applications 53 (1) (2012) 207-226.

[28] J. S. Coleman, Introduction to mathematical sociology, London Free Press Glencoe.

${ }_{380}[29]$ L. C. Freeman, C. M. Webster, D. M. Kirke, Exploring social structure using dynamic three-dimensional color images, Social Networks 20 (2) (1998) $109-118$. 